Tohoku J. exp. Med., 1967, 92, 265-270

\title{
Color Isodose Curve Plotter Applied to Beam Focus Radiography*
}

\author{
Sadayuki Sakuma \\ Department of Raliolog!y (Prof. S. Takahashi), \\ Nagoya University School of Medicine, Nagoya
}

\begin{abstract}
An apparatus was construted to facilitate superimposition of the beam focus radiogram upon the axial transverse tomogram, in order to guide subsequent radiation therapy more accurately. The apparatus antomatically draws six isodose curves in three colors, by plotting the relative doses of the beam focus radiogram. In addition, the isodose chart was made at the same magnification as that of the axial transverse tomogram. A satisfactory and reliable technique was achieved. The accuracy and the reproducibility of the apparatus was good. Clinical application of this method to a case of hypophyseal tumor is described.
\end{abstract}

The beam focus radiogram ${ }^{2-7}$ has been studied by superimposing it upon the axial transverse tomogram. By this method, however, the beam focus image becomes sometimes too indistinct to give accurate information, because of the subtraction phenomenon of the image of the axial transverse tomogram and that of the beam focus radigoram. ${ }^{7}$ In order to avoid this trouble, the author attempted to use the isodose curves obtained from the beam focus radiogram in place of the original radiogram as described below.

\section{Method and Apparatus}

The beam focus radiogram has been obtained with an exposure dose of about $12 \mathrm{R}$ at the rotation center of the film, ${ }^{4,6,7}$ as the linear portion of the characteristic curve of the film lies in the range from $7 \mathrm{R}$ to $20 \mathrm{R} .^{5}$

A Narumi microphotometer, type 250 , was reconstructed so that the lighter attached to the recording pen ran along the scale bar and facing the lighter, and six appropriately placed microphotocells were attached to the photometer (Figs. 1 and $2)$. The beam focus radiogram was placed on the glass plate over the light of the photometer, and the glass plate was moved so as to be scanned by the photodetector (1) in Figs. 1 and 2. The light passing through the radiogram was converted to electric current. According to the intensity of electricity, the lighter at the recording pen (2) changed its position along the bar and lighted one

Received for publication, March 11, 1967.

* Studies on beam focus radiography, 4 th report.

This paper was read at the 31 th meeting of Chubu Radiological Society, June 24, 1966.

The research work was made by a grant in aid of the Japan Ministry of Education. 


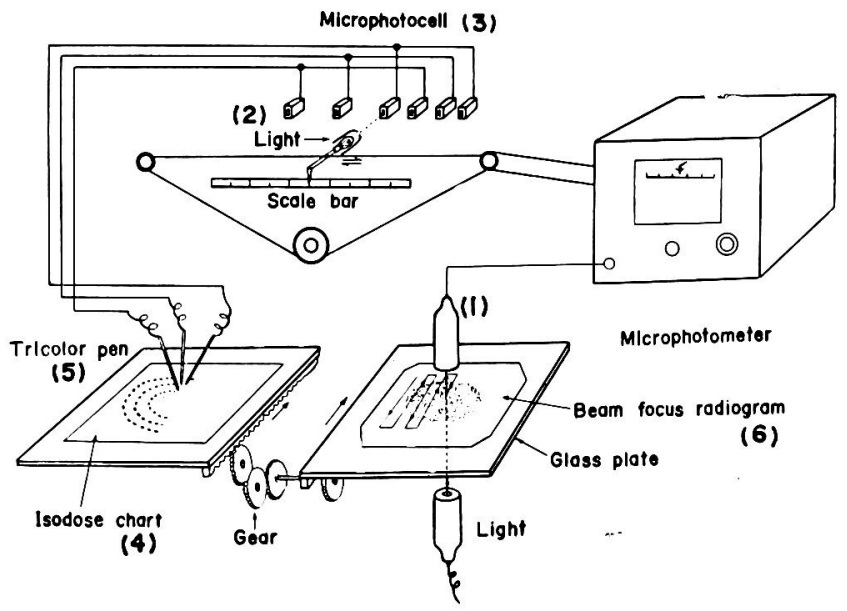

Fig. 1. Schema of the apparatus.

Fig. 2 A

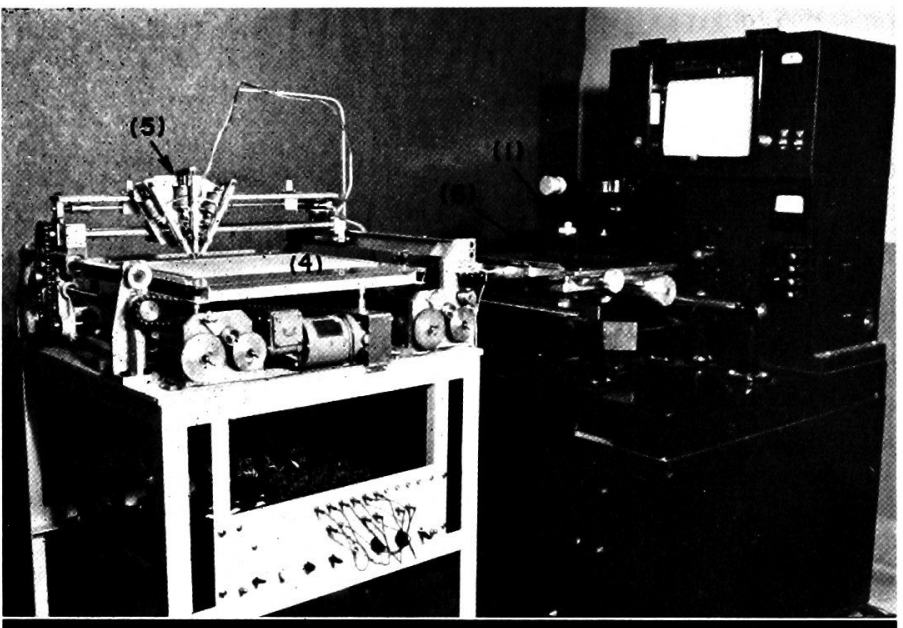

Fig. 2 B

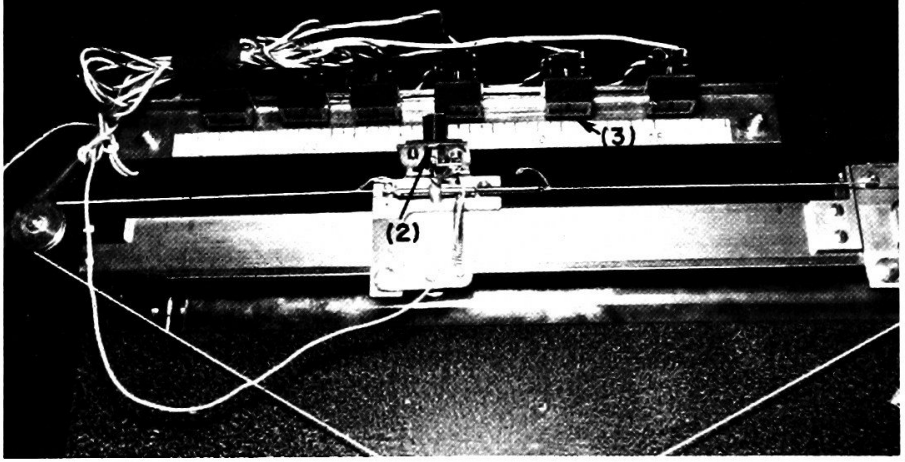

Fig. 2. A, B: Over-all view of the apparatus. (1): Photodetector. (2): Lighter. (3): Six microphotocells. (4): Isodose chart on the isodose curve drawing desk. (5): Tricolor recording pen. (6): Beam focus radiogram on the glass plate. 
of the six microphotocells (3).

In addition, translucent recording paper was placed on the isodose curve drawing desk (4).

When, for instance, the density of the film was $90 \%$ of the center of the rotation, the lighter ( $(2)$ occupied the position of a relative dose of $90 \%$ on the bar and exposed the microphotocell placed there. The recording pen (green) (5) worked to plot a dot on the recording paper, which moved on the drawing desk in coordination with that of the beam focus radiogram on the glass of microphotometer (6). Thus the dots on the curves of $90 \%$ isolose were drawn on the translucent paper of the desk.

By means of a specially made gear, the size of the isodose curve was drawn so as to be 1.27 times of the size of the actual beam focus in the patient's body, in order to correspond with the size of the axial transverse tomogram. The distance on the scale bar of the photometer between the position of density 0 and density 1 was $10 \mathrm{~cm}$. The aperture of the slit at the front of the microphotocell and the lighter (2) were each $0.5 \mathrm{~mm}$ in width. Consequently, the maximum size of a dot was three times larger than the width of the aperture of the slit, or $1.5 \mathrm{~mm}$. Therefore, the error of plotting the dot would be 0.015 in density at the most. This is considered to be negligible in practice.

\section{Reliability of the Value in Isodose Curve}

\section{A) Talue of density obtained by means of the densitometer}

In order to know how accurately the Narumi microphotometer provides the correct value of the density of the film, the standard density wedge attached to Shimadzu-Yamabe-densitometer was measured by this photometer. The distance between the density 0 and 2.5 on the wedge was $13.7 \mathrm{~cm}$. The density curve, obtained by Narumi microphotometer was linear, showing a density range from 0 to 2.5. The shift of the recording paper of the Narumi microphotometer was 13.7 $\mathrm{cm}$. This proved that the photometer worked correctly for this purpose. It was therefore concluded that this apparatus was applicable to the present experiment.

\section{B) Work of plotting}

The following experiment was carried out to examine whether plotting was made accurately or not.

The same standard wedge as in the experiment $A$ was placed on the glass plate of a film holder, in place of the film itself. Microphotocells were attached on the bar so as to work at the film densities of $0.5,1.0$ and 1.5. When the experiment was made, the dots drawn mechanically on the plotting desk were $4.5 \mathrm{~cm}$ distant from each other. As the reduction rate of distance between dots was 1.27/1.54, compared with that of the actual distance of the beam focus radiogram, this experiment proved that the beam focus was correctly reduced.

Five repeated experiments revealed the same results. The size of the dot was $1 \mathrm{~mm}$ in diameter, which was the same in size as the dot drawn without scanning. 
From the data obtained, it can be said that reproducibility of the plotting was correctly made, and that the dot was drawn not in a dash but in a dot, when the gradient of the density was about $0.2 / \mathrm{cm}$.

\section{C) Clinical application}

By means of this apparatus isodose curves were drawn from the beam focus radiograms taken during moving field therapy or conformation therapy.

Conformation radiotherapy with the hollow-out technique to shield the eyeball was applied to a pituitary neoplasm. The patient was positioned on the treatment table so that the center of the sella turcica coincided with the center of rotation of the unit. When the exposure dose at the center of the film placed on the rotation table of beam focus radiography was estimated to be about $12.5 \mathrm{R}$, irradiation was terminated. When the density of the film was measured, it was actually $12 \mathrm{R}$ (Fig. 3A). With this radiogram, iosodose curves in the range between $90 \%$ and $40 \%$ at intervals of $10 \%$ lines were drawn on the translucent recording paper (Fig. 3B).

In order to identify the relative positions of different isodose curves, the dots were drawn with different colors. The green dotted line indicates the $90 \%$ isodose line, the red line, $80 \%$, and the black line, $70 \%$. The isodose chart was thus obtained. The $90 \%$ isodose line obtained from the beam focus radiogram by this method was located about 1-2 mm inside the one drawn from data obtained actually by ionization chambers. The isodose lines of $80 \%, 70 \%$ and $60 \%$ obtained from the beam focus radiogram coincided with those obtained by ionization chambers, while the $50 \%$ isodose line thus obtained was located about $2-3 \mathrm{~mm}$ outside one of the isodose curves obtained by measurement by means of the ionization chambers.

An example of an application of the method to clinical practice, the isodose curves obtained by this method superimposed on the axial transverse tomogram is shown in Fig. 4.

\section{Discussion}

Although the isodose curve was usually drawn by means of a phantom experiment, it is possible to draw one directly from a beam focus radiogram obtained from a patient. One can reduce the size of the isodose curve in any desired magnification ratio. The advantages of our method are that it provides a simple method of obtaining isodose curves from the radiogram and further that it provides multi-color plotting ${ }^{1}$ which is much more convenient for use than monocolor plotting. ${ }^{8}$

The procedure with this apparatus employs the substitution of the isodensity curve by the isodose curve, which bears a linear relationship to the isodensity curve. ${ }^{5,6}$ Therefore, it requires that the accuracy as well as the reproducibility of the densitometer is of a high degree. The new apparatus was satisfactory in these two points. However, there still remains a problem. The isodose eurve represents a false dose distribution as compared with that measured in the human body, since 


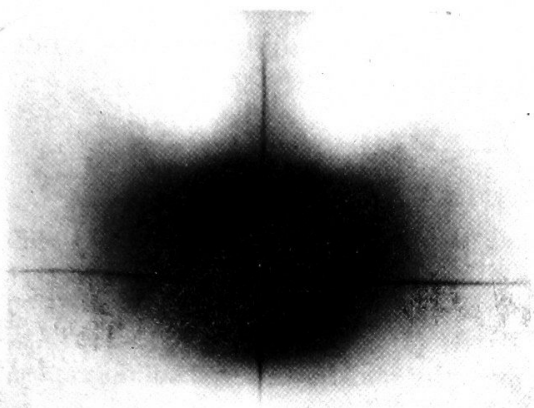

Fig. $3 \mathrm{~A}$

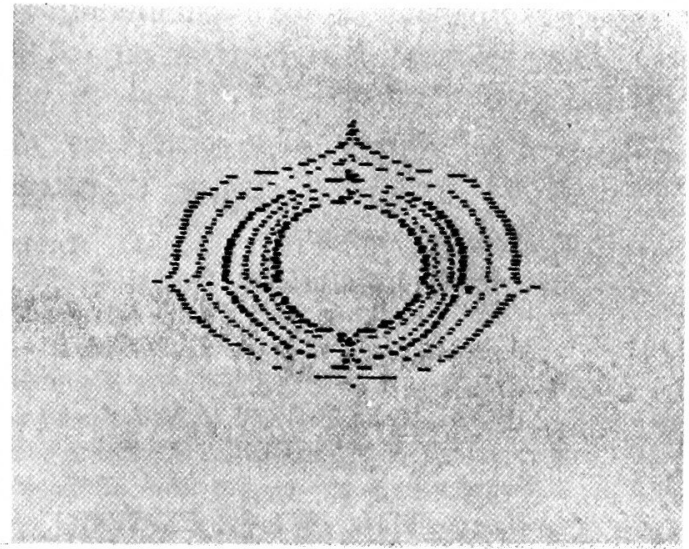

Fig. 3B

Fig. $3 \mathrm{~A}$. Beam focus radiogram taken from hollow-out technique applied to a pituitary neoplasm.

Fig. 3 B. Isodose curve taken by means of our method from the beam focus radiogram shown in Fig. 3A.

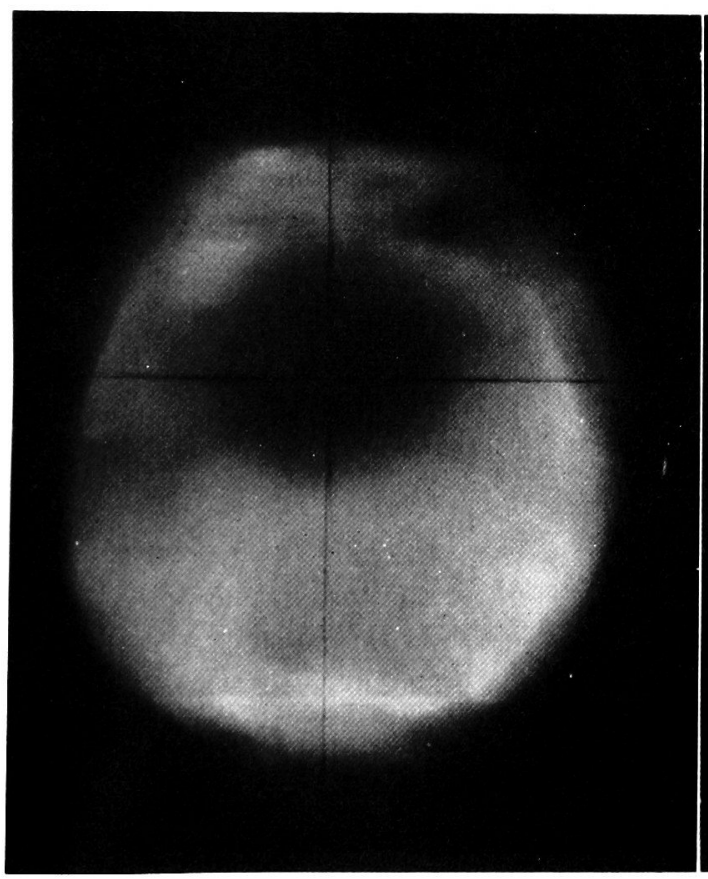

Fig. $4 \mathrm{~A}$

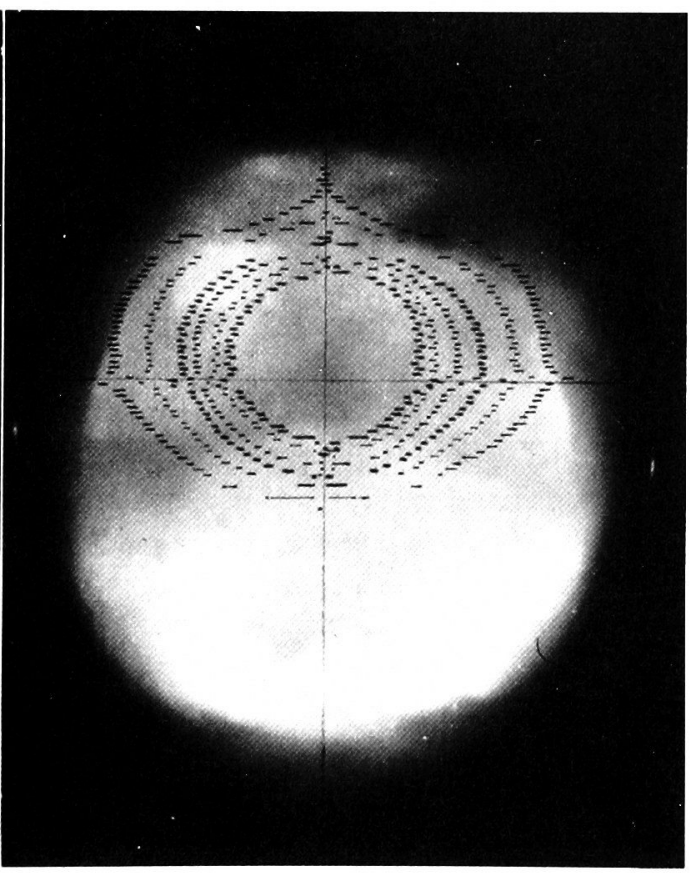

Fig. 4B

Fig. $4 \mathrm{~A}$. Beam focus radiogram shown in Fig. $3 \mathrm{~A}$. Superimposed on the axial transverse tomogram of the head.

Fig. $4 \mathrm{~B}$. The axial transverse tomogram with the isodose curve taken from beam focus radiogram. The details of the axial transverse tomogram remain visible and are not obscured, as in Fig. $4 \mathrm{~A}$ by the beam focus radiogram. 
the two parameters are essentially different. ${ }^{6}$ At any rate, the experimental result shows that the doses between 80 and $70 \%$ are correct and reliable for the clinical practice.

\section{References}

1) Matsuda, T. \& Sawada, M. A new method for taking a colour scintiscanning. Nippon Igakuhoshasen Gakhai Zasshi (Jap.), 1963, 22, 1297-1303.

2) Okajima, S. \& Sakuma, S. Measurements of dose distribution in conformation radiotherapy. Nippon Igakuhoshasen Gakkai Zasshi (Jap.), 1966, 26, 1199-1207.

3) Sakuma, S. \& Takahashi, S. Beam focus radiography and beam focus solidography. Nippon Igakuhoshasen Gakikai Zasshi (Jap.), 1962, 22, 871-876.

4) Sakuma, S.\& Takahashi. S. Beam focus radiography for taking the radiogram of the axial transverse cross section of the treated region in high density of dose. Tohoku J. exp. Med., 1965, 87, 244-248.

5) Sakuma, S. Cassette and interspacer in beam focus radiography. Tohoku $J$, exp. Med., 1965, 86, 281-291.

6) Sakuma, S. Beam focus and its image on beam focus radiogram. Tohoku J. exp. Med., 1965, 87, 306-321.

7) Takahashi, S. Conformation radiotherapy. Rotation techniques as applied to radiography and radiotherapy of eancer. Acta radiol. (Stockh.), 1965, Suppl, 242.

8) Takizawa, M. Film scanner and its application for isodose plotter and isodose synthesizer. Nippon Igakiuhoshasen Grakkai Zasshi (Jap.), 1964, 24, 510-517. 